Meta

Journal des traducteurs

Translators' Journal

\title{
Pour une physiologie du néologisme
}

\section{Pierre Widal}

Volume 18, numéro 4, décembre 1973

URI : https://id.erudit.org/iderudit/004598ar

DOI : https://doi.org/10.7202/004598ar

Aller au sommaire du numéro

Éditeur(s)

Les Presses de l'Université de Montréal

ISSN

0026-0452 (imprimé)

1492-1421 (numérique)

Découvrir la revue

Citer cet article

Widal, P. (1973). Pour une physiologie du néologisme. Meta, 18(4), 355-364.

https://doi.org/10.7202/004598ar d'utilisation que vous pouvez consulter en ligne.

https://apropos.erudit.org/fr/usagers/politique-dutilisation/ 


\section{Pour une physiologie du néologisme}

Tout ce qui est vie emprunte sans cesse à son entourage les éléments de développement et de conservation nécessaires. Même si une structure paraît fondamentalement statique, elle réclame néanmoins sans cesse quelques apports, ne fût-ce que pour remplacer les diverses parties définitivement sclérosées. Les ambiances varient sans cesse, nuit, jour, été, hiver. L'adaptation est une constante de la survie. Cela est aussi vrai pour les sapins que pour les rats, pour l'homme que pour son langage. Les modifications vont en s'accélérant et la civilisation d'aujourd'hui n'a plus beaucoup de points communs avec celle d'il y a cent cinquante ans. Bien qu'un tel truisme soit quelque peu embarrassant à énoncer, le fait est que nos arrière-grands-parents seraient littéralement affolés par la manière dont se conduit l'existence contemporaine. Ils ne le seraient pas moins par ses modes très libres d'expression. Le langage a subi l'effet des inventions, du rythme de vie, des transformations de la morale. Le théâtre du dix-neuvième siècle, d'Alexandre Dumas fils ou d'Émile Augier, traite de problèmes résolus depuis si longtemps qu'il est devenu injouable. Les romans sociaux des Goncourt ou d'Émile Zola n'excitent plus l'indignation, ils sont devenus des vignettes historiques. On peut en considérer l'objet comme périmé, demain, on dira « râpé ». L'homme n'est plus le même, ses extériorisations et ses paroles en ont subi le contrecoup.

Si l'on veut réfléchir à la physiologie d'une langue, quelques questions émergent, inévitablement, en priorité. Existe-t-il de nos jours des faits qui appellent des mots nouveaux, des définitions précédemment inconnues? Indéniablement. Des impressions ressenties différemment ou de manière inédite ? Sans doute. Des idées originales, après six millénaires ? Peut-être. Et la valeur expressive et descriptive de beaucoup de mots est-elle restée constante? Assurément non, l'érosion étant une donnée permanente et son accélération flagrante.

C'est alors que se pose le problème du néologisme, de sa valeur et de son opportunité. Ce phénomène du «parler nouveau», si l'on veut aller trop loin, 
a commencé avec le premier grognement d'hominidé de celle des branches qui a réussi. Sans remonter à des origines aussi peu explicites, il est de fait qu'on rencontre le néologisme à tous les âges et à tous les niveaux de culture. En un certain sens, il est une donnée fondamentale de développement, mais il est parfois difficile de le situer ou de le classer avec précision. Le latin est devenu du bas latin. À quel moment peut-on se croire en droit de ne plus parler d'abâtardissement, de néologisme grammatical et de tenir pour mot nouveau un phénomène qui n'est peut-être qu'une étape d'une évolution? Il y a eu des rejets espagnols, italiens, provençaux, périgourdins. Des raisons historiques ont fait prévaloir le piémontais, le castillan et, dans un certain secteur, le parler du Vexin français. Les guerres ou la distance et bien d'autres conditions ont ensuite créé des formes déviantes, des modifications particulières, aux Antilles, au Québec, voire encore en Louisiane ou dans le Val d'Aoste. À l'inverse, il existe en Alsace un village dont les habitants, groupés au sommet d'une colline, parlent exclusivement le suédois du temps de Gustave Adolphe, leurs ancêtres s'étant arrêtés là il y a trois siècles.

Même si l'évolution les a rendus quelque peu différents, les termes essentiels de la langue mère restent directement compréhensibles dans les langages mutants, déduction faite de quelques apports de caractère osmotique mineurs. Or, fait caractéristique, les transformations ont suivi une cadence que l'on peut appeler normale, si l'on se réfère au rythme séculaire, et ceci jusqu'au milieu du dixseptième siècle. Depuis cette époque, tout s'est figé, presque complètement, dans la langue mère tout au moins, pour des raisons évoquées infra. Il a fallu cependant depuis lors tenir compte de l'industrialisation, des inventions et des techniques nouvelles, de la possibilité puis de la facilité de déplacement des personnes, de la transmission courante des idées et de l'information, enfin de la nécessité de plus en plus impérieuse de la communication des techniques.

Tous les vocabulaires, partout, s'en sont ressentis. Des esprits ingénieux ont cru, avec l'espéranto et le volapuk, résoudre le problème du langage universel. Leur échec a prouvé, une fois de plus, qu'il était vain de s'attaquer à l'usage et que les initiatives théoriques les plus rationnelles achoppent, comme une vulgaire marchandise, sur des problèmes de distribution. Les jeux de l'intelligence ne constituent pas par eux-mêmes un message. L'intervention du langage des machines, snobol, cobol ou autre, est une réponse hautement spécialisée, réservée actuellement à un petit clan de techniciens, mais à laquelle l'avenir peut éventuellement ouvrir plus largement les portes des communautés.

De toute manière, l'humanité est en pleine mutation et les divers apports linguistiques se transforment en un véritable flot, sans cesse grossissant et qui est susceptible de modifier dans un avenir prévisible une importante partie du fonds général. L'invasion des néologismes en est le symptôme, comme il est aisé de le constater en comparant des éditions consécutives d'un lexique ou d'un dictionnaire.

Le néologisme est, bien entendu, toujours éphémère, au sens étymologique du mot. S'il est provisoirement accepté, puis rejeté, il tombe dans l'oubli et devient incompréhensible. C'est là une question de mode, donc d'oubli. Le mot «urf 》, courant en 1900 au point d'avoir été d'usage fréquent dans les journaux à clientèle cultivée du temps, le Gil Blas, voire le Gaulois, est incompréhensible à tous ceux 
qui ne sont pas amateurs d'un folklore rétrospectif. Par contre, «folklore » a été accepté et a passé dans le vocabulaire courant. On ne peut plus ici parler de néologisme quand les gouvernements fondent des musées folkloriques et que le terme a survécu un certain temps.

Il y a des néologismes nécessaires, généralement techniques, et d'autres qui sont simplement des synonymes, des néologismes d'ignorance, en quelque sorte, comparables aux doublets, des simplifications, des substitutions ou des déformations imposées par la structure même de certains mots. Il y a des néologismes d'invasion, amicale ou non, O.K., Verboten; des néologismes de prestige, les jockeys et les grooms d'avant-hier; des néologismes de jactance, Ciao, Bye Bye; enfin et surtout des néologismes de consommation ou d'actualité, « radio », « télévision », «z.u.p. » et combien d'autres.

Il convient cependant, avant de poursuivre, de cerner avec précision les significations possibles du mot néologisme lui-même. Il a un sens étroit, celui de mot nouveau forgé de toutes pièces ou enchaînement dérivé à partir d'un auteur déjà existant, il a aussi celui dans lequel il fut utilisé lorsqu'on l'inventa dans le salon de $\mathrm{M}^{\text {me }}$ de Lambert; pour les esprits raffinés qui le composaient, il fallait du «neuf, un tour fin et serré, une manière nouvelle » de dire. Ils exigeaient des formules qu'elles fussent aussi acérées que leur intelligence. Dans cette acception, le néologisme favorise le jaillissement de l'idée, la fraîcheur des images et l'originatité du style. Il dévalorise le cliché, il affine le langage. Mais, même si cette conception très large semble de loin la meilleure, il n'en reste pas moins nécessaire d'analyser les causes qui ont provoqué la naissance du mot, d'examiner ses sources et ses chances de réussite, avant de l'enregistrer et de le voir se soumettre au purgatoire de l'usage.

Tant de mots ont été, à un moment donné, des néologismes que l'examen de leur fortune peut être instructive pour l'étude de l'évolution de la langue à des moments précis; peu importe qu'ils aient été d'abord écrits en italique ou entre guillemets il y a un siècle ou deux, l'enseignement à retirer n'en sera pas moins précieux.

Sont, bien entendu, à exclure a priori d'une pareille étude les modifications vicieuses, les prononciations incorrectes et autres formes d'abâtardissement courantes, néologismes syntaxiques ou grammaticaux de l'ordre de «se rappeler de » et que l'usage finit parfois par imposer, en vertu d'un regrettable nivellement par le bas.

Le néologisme est emprunt, résurgence ou invention. Il correspond à un besoin ou à une facilité. Depuis l'invention de l'imprimerie, de l'orthographe et de l'école obligatoire, il n'est plus que rarement attribuable, dans la transmission, à une déformation d'origine orale, incompréhension ou audition, sauf dans le cas de termes techniques compliqués, qui font fortune dans le langage général. Il s'agit alors le plus souvent de cuirs, comme «infractus » ou « aréodrome », qui ne paraissent pas devoir être acceptés dans le langage écrit, ou d'abréviations 《 auto » ou « télé »— introduites par commodité ou paresse. 
Un néologisme apparaît subitement, mais on ne peut constater son existence qu'à partir du début de sa diffusion. Pourtant, il y a eu un stade antérieur, celui de la naissance. La naissance, c'est l'adoption du mot forgé ou spontané, comme moyen de communication avec un interlocuteur. Si celui-ci en est satisfait, il l'utilise à son tour et il y a alors commencement de diffusion. On doit constater que celle-ci, au stade élémentaire, est toujours d'un type très particulier. Le mot entre dans le jargon courant d'un clan. Puis, par osmose, il peut ou non servir à la collectivité, être ou non partie du fonds reconnu.

Par exemple, une ouvrière s'exclamera devant une couture faite sans soin et qui a des fronces sur toute sa longueur : «C'est mal fait, ça griche ! » Le mot "gricher», dans la profession et chez les commerçants qui s'y rattachent, sera instantanément compris. De même, par le client qui l'ayant entendu une fois s'en servira désormais, car le son en est imagé et facile à retenir. La diffusion est donc suffisante pour que «gricher» ne soit plus considéré comme un mot de jargon ou un terme technique. Il est absolument d'usage constant, mais ne figure cependant dans aucun dictionnaire usuel. Il est parti d'un petit monde spécialisé, d'un clan. On ne saurait trop accorder d'importance à cette notion. La fortune, la survie d'un mot vont dépendre en fin de compte du prestige et de l'influence du clan. C'est, en termes de mise en marché, un problème de distribution. "Griche » n'a pas acquis droit de cité, mais les portes se sont ouvertes largement devant «brio » introduit par Stendhal avec la bénédiction de Charles Nodier, de Balzac et de quelques caricatures de Gustave Doré, Cham et Daumier.

Il faut, bien entendu, envisager très souplement la notion de clan. Il peut s'agir, aux deux extrêmes, de deux ou trois personnes ou d'un demi-continent. Ausweiss a été utilisé pendant quelques années dans la langue courante de la moitié de l'Europe ; les cavaliers de concours hippique, clan spécialisé s'il en fut, parlent affectueusement de leurs chevaux favoris en les appelant « mon saucisson ».

Le néologisme est fréquemment un emprunt. Dans un congrès, le technicien prend parfois l'interprète de court et celui-ci, placé devant un fait nouveau ou une constatation sans précédent, tenu de plus à une transmission instantanée, francise ou adopte un nom étranger pour lequel il n'y a pas à sa connaissance d'équivalent. Il s'agit souvent aussi du reste d'un néologisme dans la langue du locuteur, parfois d'un sigle verbalisé comme cela est commun dans les langages anglo-saxons. Que faire impromptu devant laser, jeep, marketing ou merchandising, Nasa ou FannyMay, voire un ordre stop loss? La périphrase est généralement difficile ou brise le rythme. La traduction écrite corrigera, si elle le peut, les fautes de l'interprétation, mais là aussi, le temps presse. Le document doit généralement être distribué dans des délais très courts, parmi beaucoup d'autres. On ne peut faire de recherches et l'on se contente au mieux d'une note explicative, en bas de page. Les spécialistes s'emparent de la formule, l'adoptent : la diffusion est commencée. La marche arrière devient sans cesse plus difficile et, bien souvent, quand on parvient à une véritable traduction, généralement plus longue et, par principe, plus compliquée, puisqu'il s'agit d'expliciter une nouveauté, le pli est pris et les utilisateurs s'imaginent trouver des nuances qui rendent le mot substitué impropre. 
Il est toujours facile de décider en théorie qu'il suffit d'élargir conventionnellement le sens d'un mot donné pour faire face au besoin nouveau et qu'on englobera désormais une acception complémentaire, mais le problème est d'en imposer l'emploi. Pour un temps, au moins, le néologisme d'emprunt entre dans l'usage.

La renaissance de mots archaïques ou désuets est un phénomène relativement fréquent. Il est difficile cependant de tracer des limites certaines. Le mot « fiable » est un très vieux mot français. Le Dictionnaire de la langue au $X V I^{e}$ siècle, de Huguet, le donne comme couramment employé, avec de nombreuses citations. Littré cite «fiable » comme archaïque, quoique parfois encore utilisé en BasseNormandie. Il ne figure plus dans l'édition du Dictionnaire de l'Académie de 1874, ni davantage dans celle de 1932. Robert n'en fait pas état, mais il reparaît dans la dernière édition du Petit Larousse, datée de 1973. (Il semble par contre être resté communément employé au Québec.)

Il renaît dans un clan très spécialisé de techniciens de l'électronique et se diffuse dans le grand public avec la publicité consacrée aux appareils sonores, «éminemment fiables 》 ou d'une « fiabilité totale ».

Or, pour le clan, fiable a été incontestablement un néologisme créé à partir du latin avec le sens étroit de «qualification d'une constance déterminée » de certaines mesures. La langue commune en s'en emparant a rendu au mot son sens ancien de "digne de confiance », et l'accole sans hésiter à un notaire comme à un magnétophone. On peut en pareil cas se demander où sont les frontières du néologisme.

Le cas n'est naturellement pas isolé et il n'est pas difficile de trouver quantité de termes qui ont eu un destin analogue.

Quant au néologisme forgé de toutes pièces, l'imagination de l'auteur, des coïncidences ou le hasard en fixent la formation. L'inventeur des premiers acides barbituriques leur donna ce nom parce que, dans la petite ville où il travaillait, il était lié d'amitié avec des officiers d'artillerie. Il fut invité au banquet de la Sainte-Barbe, le lendemain du jour où il avait réalisé cette synthèse sur laquelle il était penché depuis un temps infini. Quand il annonça sa découverte à ses amis, le nom de Barbe vint spontanément à l'esprit. Un dérivé pharmaceutique, le véronal, a été nommé ainsi en souvenir d'une remarquable nuit de sommeil passée à Vérone par son inventeur. Le mot nylon serait le sigle du cri Now, you lousy old Nippon poussé en signe de victoire par le chimiste des laboratoires Du Pont, chargé de trouver un substitut de synthèse aux soies japonaises.

Ce sont en fait les inventions qui sont la source principale des néologismes nécessaires. Quand elles sont d'origine purement scientifique, on les baptise de termes généralement empruntés au grec ancien ou on forme des noms composés, aux significations évocatrices pour les spécialistes utilisateurs. On ne peut que regretter les libertés que prennent des techniques moins ésotériques avec une étymologie qui mériterait mieux. En face des humbles «tourne-vis », dont le tiret a disparu, des chasse-goupilles, des serre-joints de l'artisan, apparaissent des mots de prestige : automobile, autorail et trolleybus, ou de mystère : informatique. Ils 
ont eu des concurrents dans des secteurs voisins, inventeurs du zoom, du reflex ou des media. Une grande partie de ce vocabulaire est assurément indispensable et l'on imagine mal quel mot classique et courant substituer avantageusement à cybernétique ou à psychosomatique. Ces néologismes-là répondent à des critères de nécessité absolue, ils sont donc sains et ne provoquent aucune réticence.

En fait, cette nécessité est la caractéristique primordiale de la diffusion. Les désignations précises sont du reste moins soumises à l'érosion que la formulation des sentiments. Les émotions sont plus fragiles que les embrayages ou que les roses, elles appellent plus volontiers des descriptions au goût du jour. La mode joue davantage avec les élans de l'âme qu'avec les combinaisons chimiques ou les roues dentées.

Assurément, il est d'autres facteurs qui entrent en jeu. Les exigences du parler courant maltraitent souvent les mots, c'est une condition de l'évolution des langues. De même que le sens s'atténue, la prononciation rabote et assouplit. Délectuaire est devenu électuaire, fresc s'écrit et se prononce frais. Un élément protecteur provient de la diffusion de l'écriture. Aéroport devrait subsister devant aréoport et le reléguer dans les ténèbres extérieures. Par contre, l'acide désoxyribonucléique a reçu, dans tous les laboratoires où l'on a affaire à lui, un sobriquet ou une abréviation. De tout temps, il en a été de même partout. Les mots trop longs ou difficiles à prononcer sont malmenés. Pour combien de locuteurs « compendieusement » signifie-t-il brièvement ? On peut hélas prédire à « pécunier » un bel avenir. La publicité aidant, l'utilisateur sans cesse suggestionné va substituer souvent également un mot facile, indéfiniment entendu et parfois heureusement descriptif, au terme qui serait normal. L'image de marque est un fait nouveau dans le langage du néologisme, mais il n'y a après tout aucune raison linguistique valable de combattre «Frigidaire» au nom de « réfrigérateur», pas plus que «Bic» en faveur de «stylographe à bille ». On ne peut pas discuter contre l'usage qui impose également certaines contractions. $\mathrm{La}$ 《voiture de location à taxamètre » de 1910 a mérité son sort. Il est tout de même préférable et plus efficace d'appeler «taxi ! » celui qui voudra bien s'arrêter ou « charger» son voyageur. Et ce néologisme-là n'est peut-être pas à rejeter non plus.

On peut, par ailleurs, aborder le problème sous un angle tout à fait différent. D'autres conditions sont en effet susceptibles de jouer et d'exercer une influence plus ou moins profonde. L'évolution de notre manière de vivre est si rapide qu'un certain nombre de données se transforment en permanence et se répercutent sur le langage. Chaque siècle ou chaque demi-siècle se croit favorisé de dons particuliers et distille sur ses propres qualités un vocabulaire spécial. Il fallait l'admirable bon sens du dix-huitième siècle, ce temps d'étincelante lucidité, pour ne pas s'élancer sur les traces du salon de madame de Rambouillet où l'on tricotait des vers alambiqués sur des pensées qui ne l'étaient pas moins. Il fallait être un Molière, un La Fontaine ou un La Bruyère pour résister au torrent qui entraîna même parfois Corneille. Il fallait être aussi sans doute Colette, Gide ou François Coppée pour ne pas se laisser séduire par les «coruscants» de Jean Lorrain, les « agenouillements de cornettes frémissantes » des Goncourt ou les délires de Péladan. 
On se demande avec stupeur à quoi correspondent ces éclosions périodiques, ces bourgeonnements de mots insolites, néologismes au moins par le «ton » et la « nouveauté ». Il faut reconnaître en tout cas qu'il n'y a là rien de « fin ni de serré » et c'est sans doute une des raisons pour lesquelles tant de ces apports n'ont pas survécu, les conditions posées par madame de Lambert et Fontenelle n'étaient pas toutes remplies. Le jargon pseudo-scientifique de notre temps ne devrait pas, par suite, échapper aux brocards du siècle prochain et, au niveau du style, le problème ne se pose pas avec moins d'acuité. L'orientation des écrivains a subi une de ses mutations périodiques. L'impact de Céline, l'effervescence surréaliste en sont des preuves perceptibles. On est bien loin de la recherche du trait et de la chute qui firent jadis la loi. Or, la véhémence, comme tous les romantismes, enrichit les littératures. On revient vers l'expression brutale de tendances de moins en moins refoulées. Il est probable qu'avec la débandade d'un code moral plurimillénaire et la débâcle des contraintes du dix-neuvième siècle, nous assistons à un retour vers des sources plus fécondes, plus naturelles et qu'il peut en résulter une nouvelle façon d'écrire, donc de fenser, donc de s'exprimer. Le parler le moins châtié entre petit à petit dans la langue policée qui l'avait jusqu'à présent repoussé.

Deux courants complémentaires se font également sentir : ils sont l'un et l'autre d'origine économique. Nos sociétés sont, pour la première fois, délivrées de certaines anxiétés primordiales, car il est de fait que l'on ne meurt plus qu'exceptionnellement de faim et de misère dans les pays occidentaux. Les plus défavorisés sont pratiquement assurés de trouver un certain secours contre le dénuement absolu et la maladie. De ce chef, avec des salaires croissants, chacun est de plus en plus exigeant sur le chapitre des avantages concrets et des agréments matériels immédiats. Comme, pour la plupart des humains, ces avantages sont doublement appréciables si l'on peut en faire étalage, il a bien fallu nommer ces succès. La magie du verbe permet une ostentation facile à peu de frais. On cherche spontanément, par suite, à se raccorder au prestigieux vocabulaire scientifique ou à des définitions pleines de gloriole. On avait vu dans un passé relativement récent les jardiniers devenir horticulteurs et pépiniéristes. Ils avaient au moins l'excuse de s'arroger des spécialisations. Mais l'artiste capillaire, virant au capilliculteur, se croit bien au-dessus du coiffeur, le préposé-à-la-distribution du courrier, en abrégé préposé, respire de ne plus être facteur et les employés de maison, ne sachant pas encore le latin, sont bien soulagés de ne plus s'appeler domestiques. Ce genre de néologisme, absurde fruit de la vanité, est haïssable, car, offrant tous les caractères du doublet et $\mathrm{du}$ synonyme, il est à la fois inutile, prétentieux et dérisoire. On peut essayer de se consoler en pensant que la vie de ce genre de mots est souvent brève, qu'ils sont adoptés par un clan qui ne parvient généralement pas à les diffuser, qu'ils sont objets de mode, donc se périment vite pour ne survivre que dans des jargons syndicaux ou administratifs à usage exclusivement interne.

Un second facteur intervient indirectement, mais non sans effet. On n'attaque plus guère les voyageurs sur les routes ou dans les auberges, tout au moins les armes à la main, et cette sécurité, somme toute généralisée, a par contraste donné naissance à un romantisme de la violence. Les récits délassants se situent dans des milieux de préférence mal connus du lecteur moyen, dans des ambiances imagi- 
naires ou tout au moins assez dépourvues de plausibilité pour le déconcerter et le dérouter, donc pour l'entraîner à admettre des narrations systématiquement extravagantes. Le client de masse dévore ces œuvres-là avec volupté, mais les rédacteurs de ces épopées savent que pour priver encore davantage leurs fidèles de toute connection avec un cadre reconnaissable, il faut les noyer dans un fatras technologique et, pourvu que le sujet s'y prête, insérer dans les dialogues des propos tenus dans une langue étrange, mystique et familière : l'argot.

Il est rare que l'argot imprimé soit authentique, car il serait alors totalement incompréhensible. Celui des textes est un mélange de mots grossiers attendus, voire exigés, et de formules pleine de vivacité. Il faut, pour réussir, l'imagination débridée d'un Frédéric Dard dont les images sont d'une extraordinaire et effervescente nouveauté. C'est là le travail essentiel des auteurs, soit qu'ils les aient inventées, soit qu'il les aient entendues tout simplement jaillir des lèvres d'un crocheteur du Port au Foin, de Gavroche ou d'un chauffeur de taxi en colère.

La formule dévastatrice d'un conducteur de camion à un piéton mécontent : "Tais-toi, mauviette, ou je téléphone à ta veuve », a un caractère homérique indiscutable. Elle est l'explosion spontanée d'un verbalisme vivace, né du tréfonds d'un langage populaire en mutation permanente. L'idée est condensée, le «tout fin et serré ». Tout est dit en un minimum de mots. C'est le néologisme d'image, le bon, celui qui explose dans l'ambiance morne des clichés. Feu d'artifice permanent de la verdeur élémentaire et consigné dans un livre, il fera ou non carrière. Ses chances, au départ, sont égales à celles de mille autres. Parfois aussi, un seul mot passera ou non la barrière. Dans le Rififi chez les hommes - et rififi a eu quelque temps droit de cité - - , Le Breton dépeint le peu d'envie d'un avare à sortir de l'argent de ses poches en disant : «Il a des oursins plein les fouilles. » Le mot fouille est imagé, il a aussi peut-être un petit avenir, et, par ailleurs, quel bel exemple de tétrasémie, selon qu'il est employé par le voleur en question, un archéologue, un douanier ou un maçon! Le premier seul, en transposant de l'argot, peut enrichir la langue.

L'argot ne passe en réalité dans le fonds commun qu'avec difficulté, car il lui faut être diffusé par des transfuges, qui trahissent la loi du secret. L'argot se veut incompréhensible pour l'étranger au clan. Il crée sans cesse, par un mécanisme d'auto-protection, des mots nouveaux, spontanés, tels le «doul » et le «doulos» le chapeau et son porteur, au sens propre comme au sens figuré de responsable (autre néologisme), des mots empruntés ou imagés « la chnouf », ou encore transposés, comme l'or appelé «jonc» par adoption de la description technique de certaines alliances par les bijoutiers. Il s'agit toujours de dissimuler le sens des communications aux non-initiés, précaution qui date de tous les temps, transmise en littérature par Villon, par Vadé et actuellement par certains romans «noirs » qui s'accompagnent d'un glossaire. Encore là, pour des considérations de vente, les phrases sont-elles très épurées, pour ne pas lasser le lecteur, et le rédacteur donne normalement priorité au pittoresque de l'image, au détriment du mot.

La diffusion se fait lentement, comme à contrecœur. Le cheminement s'étend d'abord à travers le parler le plus vulgaire, puis par nappes successives gagne en 
dernier lieu la langue écrite qui commence généralement par s'en excuser sous le paravent des guillemets et des italiques avant de procéder à la pure et simple annexion.

Le phénomène a d'autant plus d'importance qu'il ne s'agit pratiquement jamais d'une adjonction. Un mot nouveau, surtout de cette origine, est plein de vigueur, particulièrement au point de départ. Il frappe d'autant plus qu'il est encore peu connu. Il vient doubler un mot d'usage constant et, de ce fait, accélère son érosion, même s'il n'aboutit pas à son élimination. L'ennui a pu être mortel au temps de Racine, et ses héroïnes en périr effectivement. Ces mêmes clameurs qui faisaient hier trembler les loges, colères de Camille, plaintes de Bérénice ou fureurs de Roxane, ne sont plus aujourd'hui pour nous que des formules de mécontentement et, malgré tous les efforts d'un auditeur cultivé, conscient et attentif, la résonance en est à jamais perdue. Ce qui détruisit Phèdre nous sert à définir un livre soporifique ou un concert sans relief. Balzac n'osait pas écrire « embêtant », mais nos contemporains usent sans réticence d'équivalents scatologiques. Il ne reste plus grand-chose de l'anatomie humaine qui puisse rénover la terminologie usuelle. Le langage courant en trouve cependant, et la littérature américaine contemporaine les utilise sans y prendre garde autrement, depuis qu'elle a libéré la sexualité. Par un juste retour cyclique, la science a annexé l'anxiété et l'angoisse et, vers l'an 2000, ce sera peut-être là le fin du fin des superlatifs.

En résumé, il semble bien que le néologisme doit être une innovation de caractère linguistique, ou intellectuel, ou technique, nécessaire, ou bien une réaction contre l'érosion. Il naît dans un clan, pour lequel il correspond à un besoin, se diffuse avec le rayonnement du groupe, pourvu qu'il soit facile à énoncer et qu'il plaise. S'il n'a pas de cause technique, sa vie est très fréquemment brève, car il dépend de la mode. Emprunt, adaptation, invention, il a eu de tout temps une destinée imprévisible.

Cela est toutefois peut-être moins vrai maintenant. L'évolution des vocabulaires se heurte après six millénaires, dans tous les pays, à un fait nouveau, qui est susceptible d'en bouleverser toutes les données : l'invention de l'imprimerie et sa suite logique, la possibilité généralisée de lire, possibilité devenue réalité, comme la disparition des tambours de ville en est la preuve. La codification des mots, avec ses corollaires, la grammaire et l'orthographe, a porté un coup quasi mortel à la vie des langues. Il existait auparavant des mécanismes modificateurs qui servaient de soupapes de sûreté, et la lenteur de la diffusion agissait comme un frein compensateur. La disparition de l'analphabétisme et l'expansion de la presse ont bloqué tout cela et figé presque définitivement les langues vivantes. On peut à toutes fins les considérer comme quasi mortes et, à travers les formules des grands écrivains, les contempler dans un musée. Rabelais devait assez mal comprendre le sénéchal de Joinville et Voltaire, Rabelais, mais nos contemporains ne commencent à peiner que sur les textes des prédécesseurs de Corneille. En trois cents ans, mis à part un lent mais indiscutable affadissement des termes, il n'y a pas eu de modifications verbales, grammaticales ou syntaxiques majeures. La diffusion de la culture a cristallisé la langue. En reconnaissant le fait, il faut se demander si le point de 
perfection est atteint et, s'il l'est, est-ce le parler de Laclos ou celui de Flaubert qu'il faut accrocher à la cimaise?

Le néologisme, ironique et insidieux, recouvre tout doucement ces fresques préhistoriques. Moins bien armés que les paléontologues de Lascaux, les observateurs des langages savent qu'avec ses lois et ses commodités il a pour lui le temps. S'il est une pollution, il est cependant aussi une forme de vie.

PierRe Widal 